die in bezug auf Luft stark ,uiberfettet" sind, wird die Kompressionsfestigkeit von Toluol und i-Oktan in Mischung mit Sauerstoff stark erniedrigt. Die richtige Deutung dieser Verhältnisse muß weiteren Arbeiten vorbehalten werden.

Fïr die Versuche standen Mittel der Deutschen Forschungsgemeinschaft und der Reichsstelle fuir Wirtschafts. ausbau zur Verfügung. Außerdem haben wir Herm Prof. O. KRAEMER, Karlsruhe, für seine Hilfe bei konstruktiven Fragen, sowie Herm Prof. L. EbERT, Karlsruhe, für seine Beratung und ständige Hilfsbereitschaft zu danken.

Karlsruhe, Institut für Physikalische Chemie und Elektrochemie der Technischen Hochschule, den 13. Februar I939. Ularich v. Weber. Adolf RastetTer.

\section{Meeresgeologische Veränderungen in den Gewässern um Sylt (Nordsee) seit $35^{\circ}$ Jahren.}

(Meeresgeologische Arbeiten Kiel-Kitzeberg, Nr. $33^{1}$.)

Auf Anregung der Meeresgeologischen Forschungsstelle Kitzeberg bei Kiel habe ich untersucht, welche Veränderungen der Küsten und des Meeresbodens der Gewässer um Sylt in historischer Zeit stattgefunden haben.

Die in der Meeresgeologie bisher geuibten Methoden wurden für diesen $Z$ weck als unzureichend erkannt. Für Untersuchungen erdgeschichtlicher Art ist die zur Debatte stehende Epoche zu kurz. Eine Deutung von Bohrungen findet ihre Grenzen darin, $d a B$ fast alle Proben in den für diesen $Z$ weck in Frage kommenden Schichten lediglich aus Dïnensand bestehen, so daß die geringe Strukturänderung der Bohrproben keine Veränderung der Ablagerungsbedingungen beweist. Die Möglichkeit, aus genauen Messungen der Strömung und der Sandwanderung Rückschlüsse vom heutigen Zustand auf den Ablauf in den letzten Jahrhunderten zu ziehen, scheidet aus, weil der durch derartige Messungen erfaßte Zeitraum von wenigen Jahren viel zu kurz ist, um eine Erweiterung seiner Beobachtungsergebnisse aut Jahrhunderte zu rechtfertigen. Andererseits erlaubt die Kenntnis der EntwickIung in größeren historischen Zeit räumen Rückschluisse auf die künftige Entwicklungstendenz.

Als geeignete Methode erwies sich der Vergleich der erreichbaren und in gewisser Hinsicht als zuverlässig erkannten Seekarten der letzten 350 Jahre, gegebenenfalls unter Hinzuziehung nautischer Angaben aus den See(-hand-)buichern dieser Zeit. Derartige Vergleiche ließen sich infolge der Ungenauigkeit der alten Seekarten bisher kaum über die Zeit der modernen Peilkarten hinaus durchführen. Es erwies sich zur Heranziehung älterer Karten als notwendig, den Einfluß der Mißweisung klarzustellen. Von über 5o Karten und Seebüchern der Sylter Gewässer aus der Zeit von I54 bis jetzt konnten iiber 20 als geeignet angesehen werden, um durch ihren Vergleich zum Ziel zu kommen. Die wichtigsten der verwendeten Karten und Seebuicher sind: HAEYEN I585, WAGHENAER I585/88, JANSZOON I608/20, Biaeuw I627/38, Meyer I648 (Landkarte), Hoffmann i668 (Landkarte), SÖFRENSEN I695, RENARD I745, PoulseN I777, I. M. ScHMidT I $80 \mathrm{I}$, HoLst und TUXEN I807, sowie englische und deutsche Admiralitätskarten seit der Mitte des I9. Jahrhunderts. Ein Großteil der Quellen älterer Zeit ist holländischer und dänischer Herkunft.

Die wichtigsten Ergebnisse der Arbeit sind die folgenden:

I. Das heutige Vortrapptief war zwischen Sylt und Föhr bis etwa I620 durch eine Sandbank gesperrt, so daB das Wattenentwässerungsgebiet der Lister Tiefe bis um diese Zeit erheblich weiter nach Süden reichte und erst ab etwa I620 im südlichen Teil an das heutige Vortrapptief angeschlossen wurde. Im Zusammenhang hiermit ist noch lange nach I620 ein breites und tiefes Wattfahrwasser östlich Sylt nachweisbar, welches ehemals die Entwässerung einer so großen und sich so weit nach Süden erstreckenden Wattfläche durch das Lister Tief ermöglichte, nach dem Vordringen des Vortrapptiefs aber an Bedeutung verlor.

2. Füir das Vorhandensein von großen Wattflächen und gar von. Marschland westlich Syit findet sich seit 1585 kein Anhaltspunkt.

3. Die ehemalige Haupteinfahrt des Lister Tiefs ist mit der heutigen Haupteinfahrt nicht identisch, sondern verlief nördlich des heutigen Rüstsandes. Sie hat sich gleich dern

$1 \mathrm{Nr}, 32$ ist: E. WASMUND, Flachseebeobachtungen be Sturm-Niedrigwasser an der gezeitenlosen Kieler Förde (Ostsee). - Geol. d. Meere u. Binnengew. 3, 3 (x939). früher zwischen dem Haffsand und der Insel Röm hindurchgehenden Haffsandtief nach Norden verschoben. Das alte Haffsandtief ist dabei um I770 durch Anlagerung des Haff, sandes an Röm verschwunden. Das alte Lister Haupttief ist etwa seit der gleichen Zeit flacher geworden und wurde schlieBlich durch das heutige Haupttief abgelöst, welches jedoch erst um etwa ryoo entstanden ist. Das Landtief weist die geringsten Veränderungen in seiner Lage zu Sylt auf.

4. Westlich Listland ist noch 1585 eine durchgehende schmale Bank nachweisbar, welche mit der durch KiELHoLTs Notizen bekanntgewordenen Limonitsandsteinbank identifiziert wird und Rust heißt. Um I600 wird diese Bank durch die See zerschlagen. Der südliche Teil verschwindet, während sich aus dem nördlichen Teil die heute Listland vorgelagerten Sände entwickeln.

5. Es wird nachgewiesen, $\mathrm{daB}$ die Nordkante Listlands von 1585 bis heute um etwa I Seemeile $=I, 852 \mathrm{~km}$ nach Norden gewandert ist. Die Nordkante Sylts oder das Sïdufer des Lister Tiefs lag damit damals in der Gegend des heutigen Sandes Uthörn. Dieser wird als Rest eines ehemaligen Ellenbogens erkannt. Dieser Ellenbogen ist als Ganzes nordwärts gewandert und hat Uthörn dabei zurückgelassen.

6. Der Königshafen ist nicht durch den Ellenbogen entstanden, sondern der Ellenbogen durch den Königshafen. Dieser ist erst nach 1638 nachweisbar und ist als Wirkung einer wahrscheinlich durch die Sturmflut von I634 hervorgebrachten Uberflutung anzusehen. Der Ellenbogen ist somit keine Nehrung, sondern der Rest der alten Nordküste Listlands, in das der Königshafen hineingeflutet ist.

Die Begriundung dieser Ergebnisse wird in einer ausfuibrlichen Abhandlung in nächster Zeit folgen.

Kiel, den 23. Februar x 939 .

F. ZAUSIG.

\section{Zah1 der vitalfärbbaren roten Blutzellen in Vitamin A-armen Ratten.}

In jungen Ratten von etwa roog Gewicht schwankt die Zahl der roten Blutzellen in den Grenzen 5-8 Mill. $/ \mathrm{cmm}$. Ernährt man junge Ratten Vitamin A-frei, so tritt nach etwa 30-40 Tagen der bekannte Wachstumsstillstand ein, nachdem die Tiere ihre Vitamin A-Reserven zum größten Teil verioren haben. Setzt man nun der synthetischen Grundkost Vitamin A oder Carotin (Provitamin A) zu, so stellt sich schon nach wenigen Tagen die Gewichtszunahme

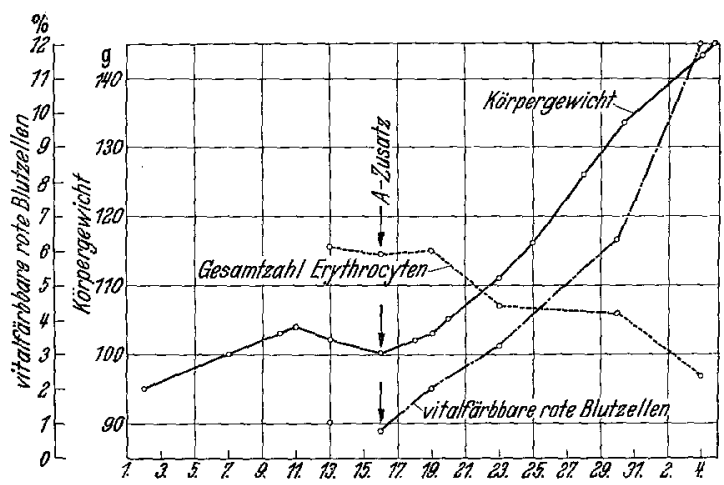

wieder ein und wird nach Tagesdosen von I-2 $\gamma$ Vitamin wieder normal, d. h. die Ratten nehmen jeden Tag um etwa $0,8-1,0 g$ an Gewjcht zu.

Untersucht man nun das Blut der Vitamin A-frei ernährten Ratten, so zeigt sich, daß zwar die Gesamtzahl der roten Blutzellen nicht sehr stark verändert worden ist - eine leichte Steigerung von etwa I $_{5} \%$ der gesamten Erythrocytenzahl macht sich bemerkbar --, daß aber die Zahl der vitalfärbbaren Erythrocyten ( $\mathrm{fEE}$ ) auBerordentlich stark abgenommen hat. Man findet nämlich bei der A-Avitaminose der untersuchten Tiere höchstens $1 / 8$ des normalen Wertes.

Gibt man den so behandelten Ratten Vitamin A-Zusätze zur Nahrung, so setzt gleichzeitig mit der Zunahme ihres Körpergewichtes eine kräftige Zunahme der Zahl der vitalfärbbaren Erythrocyten, VfE., ein; sie erreicht nach 2 bis 3 Wochen die normale Höhe und geht oft über diesen Normalwert hinaus (Figur). 